\title{
Weighted Bootstrap Approach for the Variance Ratio Tests: A Test of Market Efficiency
}

\author{
Dilip Kumar \\ Indian Institute of Management Kashipur, Kashipur, India \\ Email: dilip.kumar@iimkashipur.ac.in, dksic212@gmail.com
}

Received 30 March 2016; accepted 31 May 2016; published 3 June 2016

Copyright (C) 2016 by author and Scientific Research Publishing Inc.

This work is licensed under the Creative Commons Attribution International License (CC BY). http://creativecommons.org/licenses/by/4.0/

(c) (i) Open Access

\begin{abstract}
By means of Monte Carlo experiments using the weighted bootstrap, we evaluate the size and power properties in small samples of Chow and Denning's [1] multiple variance ratio test and the automatic variance ratio test of Choi [2]. Our results indicate that the weighted bootstrap tests exhibit desirable size properties and substantially higher power than corresponding conventional tests.
\end{abstract}

Keywords

Monte Carlo Experiment, Weighted Bootstrap, Variance Ratio, Return Predictability

\section{Introduction}

The foundation of the efficient market hypothesis lies in the ground-breaking works of Bachelier [3], Cootner [4], Samuelson [5] and Fama [6]. According to the efficient market hypothesis, the current level of the asset price fully reflects all available information, so no extraordinary gain can be made with publicly available information which directly points to random walk or martingale hypothesis. The study of the efficiency characteristics of the market impacts the regulatory framework, as well as the evolution of the market in terms of transparency and disclosures. It has policy implications which can help policy makers and regulators take steps towards financial innovations and economic development.

The existing literature provides several methods to investigate whether a given time series is a martingale or not. The variance ratio test is one of the most commonly employed procedures to study this property of the time series. The Lo and Mac Kinlay's [7] individual variance ratio test and its multiple variance ratio variant, as proposed by Chow and Denning [1], are widely used to test the martingale behaviour of the time series. These tests 
are asymptotic in nature and so can give rise to misleading results in small samples. Choi [2] proposes the automatic variance ratio test, spectral domain tests and average exponential tests to test the weak form efficiency of US real exchange rates. Wright [8] proposes a nonparametric variance ratio test based on the ranks and signs and Belaire-Franch and Contreras [9] use the principle of Chow and Denning's [1] approach on Wright's [8] individual rank and sign tests and develop joint nonparametric variance ratio tests.

In this paper, the weighted bootstrap procedure is proposed as an alternative to improve the small sample properties of the Chow and Denning [1] multiple variance ratio test and also the automatic variance ratio test. The weighted bootstrap is a resampling procedure which is applicable to data with conditional heteroskedasticity and provides a better approximation to the sampling distribution of the statistics concerned.

Section 2 presents the methodology used in this study. Section 3 presents the results of the Monte Carlo experiments. Section 4 provides conclusion of the study.

\section{Methodology}

\subsection{Variance Ratio Test}

Suppose $P_{t}$ is an asset price at time $t$, where $t=1, \cdots, T$, and $x_{t}$ be $\ln \left(P_{t}\right)$, the $\log$ price series. The first order autoregressive model is given by:

$$
x_{t}=\mu+\varnothing x_{t-1}+\varepsilon_{t}
$$

where $\mu$ is an arbitrary drift parameter and $\varepsilon_{t}$ is a random disturbance term. The random walk hypothesis (RWH) corresponds to $\varnothing=1$ and it implies that the variance of the log price increments is linear in the observation interval. It plays a very important role in testing for the weak-form market efficiency. The variance ratio test exploits the property that, if a series of asset returns is purely random, then the variance of the $k$-period return ( $k$-period differences of $x_{t}$ ) is $k$ times the variance of a one-period return.

Suppose $y_{t}$ is an asset return at time $t(t=1, \cdots, T)$. The variance ratio for holding period $k$ is defined as:

$$
V R(y: k)=\left\{\frac{1}{T k} \sum_{t=k}^{T}\left(y_{t}+y_{t-1}+\cdots+y_{t-k+1}-k \hat{\mu}\right)^{2}\right\} \div\left\{\frac{1}{T} \sum_{t=1}^{T}\left(y_{t}-\hat{\mu}\right)^{2}\right\}
$$

where $\hat{\mu}=T^{-1} \sum_{t=1}^{T} y_{t}$. Lo and MacKinlay [7] propose the following test statistics under the null hypothesis $\operatorname{VR}(k)=1$,

$$
M(y: k)=(V R(y: k)-1)\left(\sum_{j=1}^{k-1}\left[\frac{2(k-j)}{k}\right]^{2} \delta_{j}\right)^{-\frac{1}{2}}
$$

which follows the standard normal distribution asymptotically if $y_{t}$ is a martingale difference sequence, where

$$
\delta_{j}=\left\{\sum_{t=j+1}^{T}\left(y_{t}-\hat{\mu}\right)^{2}\left(y_{t-j}-\hat{\mu}\right)^{2}\right\} \div\left\{\left[\sum_{t=1}^{T}\left(y_{t}-\hat{\mu}\right)^{2}\right]^{2}\right\}
$$

\subsection{Multiple Variance Ratio Test}

Chow and Denning [1] propose a multiple variance ratio test for the joint null hypothesis $V R\left(k_{i}\right)=1$ for $i=1, \cdots, m$. The test statistics is given by:

$$
M V=\max _{1 \leq i \leq m}|M(y: k)|
$$

The decision to reject the null hypothesis is based on the maximum of the absolute value of the individual variance ratio statistics.

\subsection{Automatic Variance Ratio Test}

Choi [2] suggests a data-dependent procedure to find the optimal value of $k$. Choi propose a variance ratio test based on the frequency domain. Cochrane [10] shows that the estimator of $V R(k)$ which uses the usual consistent 
estimators of variances is asymptotically equal to $2 \pi$ times the normalized spectral density estimator at zero frequency which uses the Bartlett kernel. Andrews [11] finds that the Quadratic Spectral kernel is optimal in estimating the spectral density at zero frequency. Choi also employs the Quadratic Spectral kernel to estimate the variance ratio. Choi's variance ratio estimator is defined as

$$
V R(k)=1+2 \sum_{i=1}^{T-1} m(i / k) \hat{\rho}(i)
$$

where

$$
\hat{\rho}(i)=\frac{\sum_{t=1}^{T}\left(y_{t}-\hat{\mu}\right)\left(y_{t+i}-\hat{\mu}\right)}{\sum_{t=1}^{T}\left(y_{t}-\hat{\mu}\right)^{2}}
$$

and

$$
\hat{\mu}=\frac{1}{T} \sum_{t=1}^{T} y_{t}
$$

and

$$
m(z)=\frac{25}{12 \pi^{2} z^{2}}\left[\frac{\sin \left(\frac{6 \pi z}{5}\right)}{\left(\frac{6 \pi z}{5}\right)}-\cos \left(\frac{6 \pi z}{5}\right)\right]
$$

where $m(z)$ is the quadratic spectral kernel. Choi [2] stated that $V R(k)$ is a consistent estimator of $2 \pi f_{y}(0)$, where $f_{y}($.$) denotes the normalized spectral density of the time series \left\{y_{t}\right\}$. Choi [2] has stated that under the null hypothesis $\left(\mathrm{H}_{0}: 2 \pi f_{y}(0)=1\right)$ the $A V R(k)$ statistic is defined as

$$
A V R(k)=\frac{\sqrt{\frac{T}{k}}[\operatorname{VR}(k)-1]}{\sqrt{2}} \stackrel{d}{\longrightarrow} N(0,1)
$$

as $k \rightarrow \infty, T \rightarrow \infty, T / k \rightarrow \infty$. The variance ratio test is a two-sided test, and its critical values are taken from both tails of the standard normal distribution. The $A V R(k)$ result holds when $y_{t}$ is IID (independent and identically distributed) with finite fourth moment [12]. The variance ratio test defined here depends on the lag truncation point (or holding period) $k$. To select the truncation point optimally Choi [2] uses Andrews' [11] method.

\subsection{Weighted Bootstrap Procedure}

The following steps define the procedure of using the weighted bootstrap on variance ratio test statistics:

1. Find normalized returns $z_{t}=\left(y_{t}-\bar{y}\right) / \sigma(y)$, where $\bar{y}=T^{-1} \sum_{t=1}^{T} y_{t}$ is the mean and $\sigma(y)=\sqrt{T^{-1} \sum_{t=1}^{T}\left(y_{t}-\bar{y}\right)^{2}}$ is the standard deviation of the return.

2. For each $t$, draw a weighting factor $z_{t}^{*}(t=1, \cdots, T)$ with replacement from the normalized returns $z_{t}$.

3. Form a bootstrap sample of $T$ observations $y_{t}^{*}=z_{t}^{*} y_{t}(t=1, \cdots, T)$.

4. Calculate the required test statistic (suppose $\operatorname{VRS} S^{*}\left(k^{*}\right)$ ), the VRS statistic obtained from $\left\{y_{1}^{*}, \cdots, y_{T}^{*}\right\}$.

5. Repeat steps 1 to 4 sufficiently many (say $m$ ) times to form a bootstrap distribution of the test statistics $\left\{\operatorname{VRS}^{*}\left(\hat{k}^{*} ; j\right)\right\}_{j=1}^{m}$.

The two tailed $p$-value of the test can be obtained as the proportion of absolute values of $\left\{\operatorname{VRS}\left(\hat{k}^{*} ; j\right)\right\}_{j=1}^{m}$ greater than the absolute value of $\operatorname{VRS}(k)$.

\section{Results Based on Monte Carlo Simulation Experiment}

To evaluate the quality of Chow and Denning's [1] multiple variance ratio (MVR) test and also the automatic 
variance ratio (AVR) test statistics, we undertake Monte Carlo simulation experiments to study their size and power properties for samples of different sizes $(N=100,500,1000)$. For MVR and MVR ${ }^{*}$ test, we set holding periods $\left(k_{1}, k_{2}, k_{3}, k_{4}, k_{5}, k_{6}\right)=(2,5,10,20,40,80)$. The following models are considered to evaluate the size properties of the tests used:

Model 1: GARCH(1,1)

$$
Y_{t}=\sqrt{h_{t}} \varepsilon_{t}=u_{t} ; h_{t}=0.001+0.09 \varepsilon_{t-1}^{2}+0.90 h_{t-1}
$$

Model 2: Stochastic volatility

$$
Y_{t}=\exp \left(0.5 h_{t}\right) \varepsilon_{t}=u_{t} ; \quad h_{t}=0.95 h_{t-1}+\epsilon_{t} ; \quad \epsilon_{t} \sim N(0,0.1)
$$

In these model, we use two types of random errors: the standard normal distribution $\left(\varepsilon_{t} \sim N(0,1)\right)$ and the Student-t distribution with 3 degree of freedom (as suggested by White (2000)). To evaluate the power properties of the MVR and AVR test statistics, we use model 3 and model 4 which take the error term from model 1 and model 2 (that is, $u_{t}$ term from model 1 and model 2 also act as error term in model 3 and model 4 ).

Model 3: AR(1) model

$$
Y_{t}=0.1 Y_{t-1}+u_{t}
$$

Model 4: Long memory (ARFIMA $(0,0.1,0))$ model

$$
(1-B)^{0.1} Y_{t}=u_{t}
$$

For all the cases, the number of Monte Carlo trials is set to 1000 and the significance level is set at $5 \%$. In the following tables for evaluating size and power properties, GARCH_N and SV_N represents model 1 and model 2 with error term from Standard Normal distribution; and GARCH_t and SV_t represents model 1 and model 2 with error term from the Student-t distribution with 3 degrees of freedom. To modify the size and power properties of MVR and AVR tests for smaller samples ( $N=100,500$ and 1000), we propose the weighted bootstrap procedure. The number of bootstrap iterations is set to 500 .

Table 1 presents the size properties of the MVR, AVR, MVR ${ }^{*}$ and $\mathrm{AVR}^{*}$ test. We find severe size distortion across all data generating processes for all sample sizes for MVR and AVR test. But even after applying weighted bootstrap procedure on MVR and AVR test statistics, we find size distortion for sample sizes of 100

\begin{tabular}{|c|c|c|c|c|}
\hline & GARCH_1 & GARCH_2 & SV_1 & SV_2 \\
\hline \multicolumn{5}{|l|}{ MVR } \\
\hline 100 & 0.020 & 0.026 & 0.025 & 0.033 \\
\hline 500 & 0.029 & 0.027 & 0.037 & 0.036 \\
\hline 1000 & 0.032 & 0.028 & 0.041 & 0.047 \\
\hline \multicolumn{5}{|l|}{ AVR } \\
\hline 100 & 0.019 & 0.028 & 0.012 & 0.006 \\
\hline 500 & 0.020 & 0.064 & 0.007 & 0.016 \\
\hline 1000 & 0.024 & 0.111 & 0.021 & 0.006 \\
\hline \multicolumn{5}{|l|}{ MVR $^{*}$} \\
\hline 100 & 0.043 & 0.032 & 0.049 & 0.026 \\
\hline 500 & 0.054 & 0.061 & 0.048 & 0.052 \\
\hline 1000 & 0.054 & 0.053 & 0.054 & 0.049 \\
\hline \multicolumn{5}{|l|}{$\mathrm{AVR}^{*}$} \\
\hline 100 & 0.057 & 0.071 & 0.052 & 0.053 \\
\hline 500 & 0.047 & 0.069 & 0.056 & 0.059 \\
\hline 1000 & 0.044 & 0.043 & 0.043 & 0.046 \\
\hline
\end{tabular}
and 500. We find the size distortion to be less of a problem for MVR ${ }^{*}$ and $\mathrm{AVR}^{*}$ test statistics for a sample size of 1000 .

Table 1. Size of the tests.

$\mathrm{MVR}^{*}$ and $\mathrm{AVR}^{*}$ represent the MVR and AVR tests with weighted bootstrap. 
Table 2 reports the power properties of the MVR, AVR, MVR* and $\mathrm{AVR}^{*}$ tests when model 3 (AR(1) model) is the alternative. We find a significant improvement in the power properties of MVR and AVR tests by the application of the weighted bootstrap procedure on the conventional tests used. When we compare the power properties of $\mathrm{MVR}^{*}$ and $\mathrm{AVR}^{*}$ test statistics, we can see that the power of $\mathrm{AVR}^{*}$ test statistic is higher than that of MVR ${ }^{*}$ test statistic for most of the cases against the AR(1) model alternative.

Table 3 presents the power properties of the MVR, AVR, MVR ${ }^{*}$ and $\mathrm{AVR}^{*}$ tests when model 4 (long memory) is employed as the alternative. We find higher power for MVR ${ }^{*}$ and $\mathrm{AVR}^{*}$ test statistics for sample size 1000. For other sample sizes, we find improvement in power properties of MVR and AVR test.

Table 2. Power of the tests against the AR(1) alternative.

\begin{tabular}{|c|c|c|c|c|}
\hline & GARCH_1 & GARCH_2 & SV_1 & SV_2 \\
\hline \multicolumn{5}{|l|}{ MVR } \\
\hline 100 & 0.082 & 0.071 & 0.067 & 0.076 \\
\hline 500 & 0.285 & 0.272 & 0.407 & 0.398 \\
\hline 1000 & 0.542 & 0.445 & 0.753 & 0.738 \\
\hline \multicolumn{5}{|l|}{ AVR } \\
\hline 100 & 0.089 & 0.105 & 0.077 & 0.060 \\
\hline 500 & 0.345 & 0.343 & 0.317 & 0.352 \\
\hline 1000 & 0.582 & 0.566 & 0.636 & 0.650 \\
\hline \multicolumn{5}{|l|}{ MVR $^{*}$} \\
\hline 100 & 0.113 & 0.073 & 0.099 & 0.091 \\
\hline 500 & 0.389 & 0.355 & 0.476 & 0.459 \\
\hline 1000 & 0.650 & 0.520 & 0.808 & 0.749 \\
\hline \multicolumn{5}{|l|}{$\mathrm{AVR}^{*}$} \\
\hline 100 & 0.157 & 0.201 & 0.185 & 0.186 \\
\hline 500 & 0.418 & 0.419 & 0.553 & 0.561 \\
\hline 1000 & 0.641 & 0.579 & 0.811 & 0.820 \\
\hline
\end{tabular}

Table 3. Power of the tests against long memory.

\begin{tabular}{|c|c|c|c|c|}
\hline Long Memory & GARCH_1 & GARCH_2 & SV_1 & SV_2 \\
\hline \multicolumn{5}{|l|}{ MVR } \\
\hline 100 & 0.128 & 0.134 & 0.151 & 0.161 \\
\hline 500 & 0.654 & 0.685 & 0.407 & 0.398 \\
\hline 1000 & 0.948 & 0.951 & 0.936 & 0.957 \\
\hline \multicolumn{5}{|l|}{ AVR } \\
\hline 100 & 0.137 & 0.144 & 0.127 & 0.145 \\
\hline 500 & 0.563 & 0.555 & 0.569 & 0.588 \\
\hline 1000 & 0.888 & 0.889 & 0.885 & 0.897 \\
\hline \multicolumn{5}{|l|}{ MVR $^{*}$} \\
\hline 100 & 0.159 & 0.167 & 0.166 & 0.166 \\
\hline 500 & 0.750 & 0.732 & 0.742 & 0.722 \\
\hline 1000 & 0.950 & 0.963 & 0.956 & 0.963 \\
\hline \multicolumn{5}{|l|}{$\mathrm{AVR}^{*}$} \\
\hline 100 & 0.257 & 0.272 & 0.285 & 0.288 \\
\hline 500 & 0.748 & 0.755 & 0.731 & 0.750 \\
\hline 1000 & 0.952 & 0.951 & 0.957 & 0.945 \\
\hline
\end{tabular}




\section{Conclusion}

In this study, we evaluate the small sample size and power properties of the Chow and Denning's [1] multiple variance ratio test and the Choi's [2] automatic variance ratio test with and without weighted bootstrap approach. The size and power properties are examined based on different sample sizes $(N=100,500$ and 1000). The number of Monte Carlo trials and weighted bootstrap iterations is set to 1000 and 500 respectively. The results indicate that the size and power properties of the multiple variance ratio test and the automatic variance ratio test with weighted bootstrap are superior to the corresponding size and power properties of the multiple variance ratio test and the automatic variance ratio test without weighted bootstrap.

\section{References}

[1] Chow, V. and Denning, K. (1993) A Simple Variance Ratio Test. Journal of Econometrics, 58, 385-401. http://dx.doi.org/10.1016/0304-4076(93)90051-6

[2] Choi, I. (1999) Testing the Random Walk Hypothesis for Real Exchange Rates. Journal of Applied Econometrics, 14, 293-308. http://dx.doi.org/10.1002/(SICI)1099-1255(199905/06)14:3<293::AID-JAE503>3.0.CO;2-5

[3] Bachelier, L. (1900) Theorie de la speculation. Annales scientifiques de l'École normale supérieure, 17, 21-86.

[4] Cootner, P.H. (1964) The Random Character of Stock Market Prices. MIT Press, Cambridge.

[5] Samuelson, P. (1965) Proof That Properly Anticipated Prices Fluctuate Randomly. Industrial Management Review Spring, 6, 41-49.

[6] Fama, E. (1970) Efficient Capital Markets: A Review of Theory and Empirical Work. The Journal of Finance, 25, 383-417. http://dx.doi.org/10.2307/2325486

[7] Lo, A. and MacKinlay, A. (1988) Stock Market Prices Do Not Follow Random Walks: Evidence from a Simple Specification Test. The Review of Financial Studies, 1, 41-66. http://dx.doi.org/10.1093/rfs/1.1.41

[8] Wright, J. (2000) Alternative Variance-Ratio Tests Using Ranks and Signs. Journal of Business and Economic Statistics, 18, 1-9.

[9] Belaire-Franch, J. and Contreras, D. (2004) Rank and Sign Based Multiple Variance Ratio Tests. Working Paper, Department of Economic Analysis, University of Valencia.

[10] Cochrane, J.H. (1988) How Big Is the Random Walk in GNP? Journal of Political Economy, 96, 893-920. http://dx.doi.org/10.1086/261569

[11] Andrews, D.W.K. (1991) Heteroskedasticity and Autocorrelation Consistent Covariance Matrix Estimation. Econometrica, 58, 817-858. http://dx.doi.org/10.2307/2938229

[12] Anderson, T. (1971) Statistical Analysis of Time Series. Wiley, New York. 